\title{
Convexity cerebral haemorrhage in the neonate: in vivo ultrasound diagnosis
}

\author{
M E I MORGAN, O J HENSEY, AND R W I COOKE \\ Regional Neonatal Intensive Care Unit, Liverpool Maternity Hospital, University of Liverpool
}

SUMMARY In vivo diagnosis of subdural or subarachnoid haematoma was made in five neonates by realtime ultrasound scanning. A combination of asphyxia and sequelae of exchange transfusion were probable precipitating factors. Persistent ultrasound abnormalities relate to quality of outcome in survivors.

Large haematomas over the surface of the brain have been noted in neonatal necropsy studies for many years. ${ }^{1}$ These haematomas may be pathologically distinguished into subdural haemorrhage, now diminished in incidence and associated with obstetric trauma; subarachnoid haematoma; and subpial bleeding. Any of these may be associated with compression and venous infarction of the underlying brain. $^{23}$ Clinically, differential diagnosis between subarachnoid and subdural blood clot is not possible, although an estimate may be made of the likely site depending on the aetiological factors. ${ }^{1}$

We describe five infants (four of whom survived) in whom haematoma over the cerebral convexity was diagnosed in the neonatal period by real time ultrasound. The natural progression of the condition was followed and interventions monitored under ultrasound control.

\section{Case 1}

A girl, birthweight $2.26 \mathrm{~kg}$, was delivered at 35 weeks' gestation by emergency caesarean section because of terminal cardiotachograph tracings. There had been severe maternal rhesus isoimmunisation requiring four intrauterine transfusions. The infant appeared hydropic at birth and her Apgar score was 3 at 1 minute. She was ventilated from birth. Cord blood showed a haemoglobin value of $3.9 \mathrm{~g} / \mathrm{dl}$ and a two volume exchange transfusion with fresh rhesus negative blood was therefore performed through an umbilical venous line within the first 3 hours. A second exchange transfusion was performed at 5-6 hours of age.

At 24 hours there was bleeding from the endotracheal tube; coagulation screening showed a prolonged thrombin ratio and thrombocytopenia.
Platelet transfusion was given. Subsequently the infant's course was uneventful. She was extubated at 72 hours and displayed no neurological abnormalities in the newborn period. Developmental assessment at 1 year is normal.

Ultrasound findings Real time cranial ultrasound scanning was performed in all infants through the anterior fontanelle using an Advanced Technology Laboratories $850 \mathrm{~A}$ sector scanner with $5 \mathrm{MHz}$ transducers as described. ${ }^{4}$ At 18 hours the appearances were normal; at 72 hours a possible right temporal haematoma was noted; and by 96 hours a right temporal mass $1 \times 1.3 \times 2 \mathrm{~cm}$ in diameter was visible without midline shift but with apparent compression of the temporal lobe. The appearance persisted unaltered for 1 week (Fig. 1); by 14 days the mass was less echodense; and by 28 days ultrasound appearances were normal and have remained so. These appearances resemble pathological descriptions of subarachnoid convexity haematoma complicating rhesus isoimmunisation.

\section{Case 2}

A girl, birthweight $3.50 \mathrm{~kg}$ was delivered at 37 weeks' gestation by emergency caesarean section because of fetal distress in labour. The mother had known rhesus antibodies, but two previous children had been unaffected. Amniocentesis had not been performed. The infant appeared hydropic, and her Apgar score was 0 at 1 minute and 1 at 5 minutes. She was intubated from birth, and ascites aspirated during initial resuscitation. Cord haemoglobin was $2.5 \mathrm{~g} / \mathrm{dl}$, therefore double volume exchange transfusion was performed through an umbilical venous line twice between 1 and 5 hours. At 30 hours, 

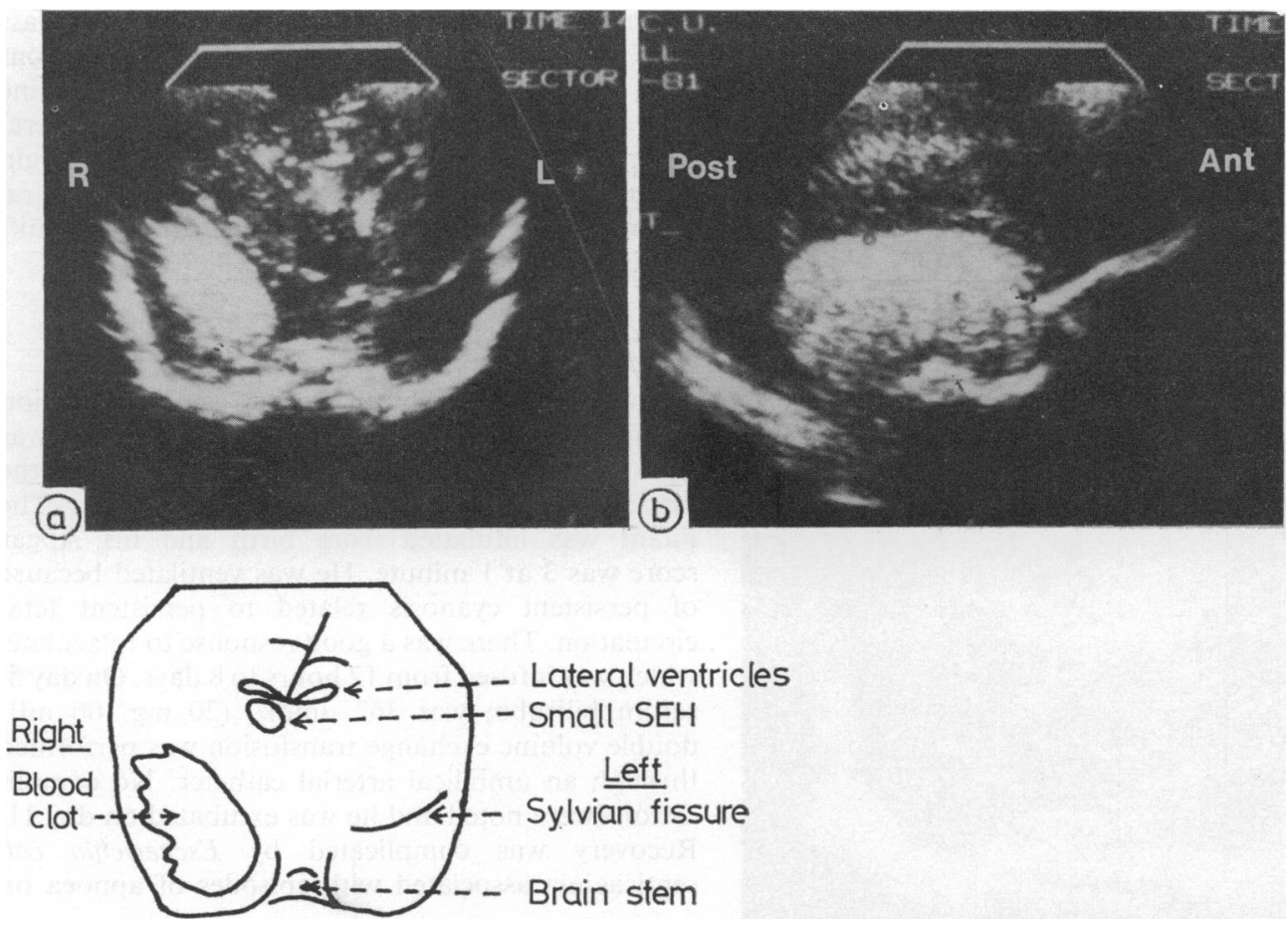

Fig. 1(a): Infant in case 1-Coronal scan showing right temporal echoreflectant mass. There is also a small right subependymal haemorrhage.

Fig. 1(b): Infant in case 1-Right parasagittal scan showing echoreflectant mass filling the site of the temporal lobe.

there was clinical collapse associated with development of bilateral pneumothoraces. After drainage, the infant was extubated. At 60 hours, pupillary inequality was noted. At 84 hours, convulsions and gastrointestinal haemorrhage occurred and the infant was transferred to the regional neonatal unit. Investigations showed thrombocytopenia though other indices of coagulation were normal. A third exchange transfusion with fresh blood was performed. Right subdural taps through the coronal suture were performed at 90 and 96 hours and 15 and $12 \mathrm{ml}$ of bloody fluid were obtained, without clinical improvement. No further fluid could be aspirated subsequently. On day 5 the infant was extubated; the right sided pupillary dilatation had resolved, and her clinical course was subsequently uneventful. At age 2 years she has a developmental quotient of about 50 and a left hemiparesis.

Ultrasound findings At 90 hours, a right sided echodense mass was noted with midline shift (Fig. 2). Over the next 48 hours, the mass became more echodense, but midline shift remained (Fig. 3). Subsequent shrinkage of blood clot occurred, with

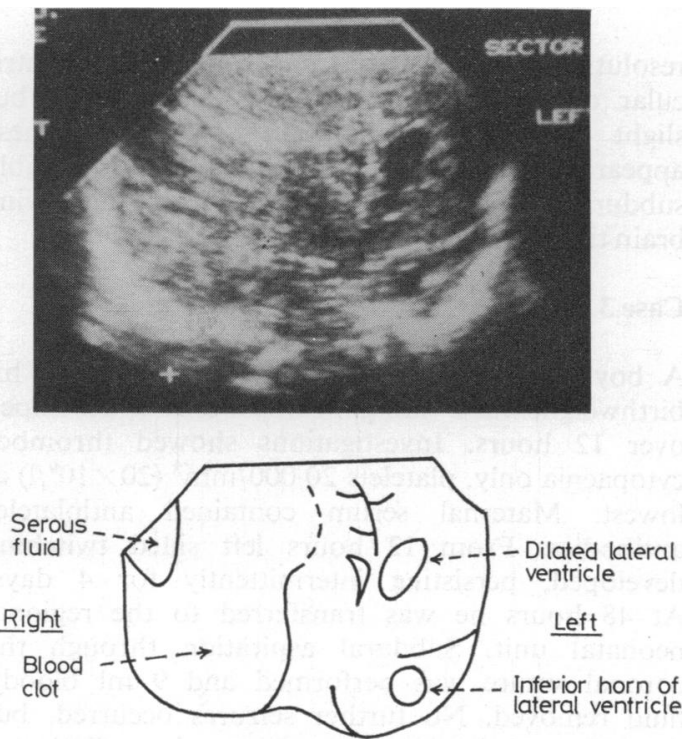

Fig. 2 Infant in case 2-Coronal scan at 90 hours showing large right sided echoreflectant mass with displacement of midline structures and dilatation of left lateral ventricle. 
Table Coagulation function before haemorrhage

\begin{tabular}{lccccc}
\hline $\begin{array}{l}\text { Case } \\
\text { No }\end{array}$ & $\begin{array}{l}\text { Platelet } \\
\text { count } \\
\left(\times 10^{9} / l\right)\end{array}$ & $\begin{array}{l}P T \\
(\text { Sec })\end{array}$ & $\begin{array}{l}P T T \\
(\text { Sec })\end{array}$ & $\begin{array}{l}\text { Thrombin } \\
\text { ratio }\end{array}$ & $\begin{array}{l}\text { FDP } \\
\mu g / m l)\end{array}$ \\
\hline 1 & $21^{*}$ & 18 & 54 & $1 \cdot 5$ & - \\
2 & $20^{*}$ & 15 & 36 & $1 \cdot 2$ & - \\
3 & $43^{*}$ & $14 \cdot 5$ & 63 & $1 \cdot 6$ & $<10$ \\
4 & $63^{*}$ & 16 & 23 & $1 \cdot 3$ & - \\
5 & $<20^{*}$ & $>60^{*}$ & $>120^{*}$ & $>3 \cdot 0^{*}$ & - \\
\hline
\end{tabular}

* = abnormal value

$\mathbf{P T}=$ prothrombin time; $\mathbf{P T T}=$ partial thromboplastin time; FDP $=$ fibrin degradation product

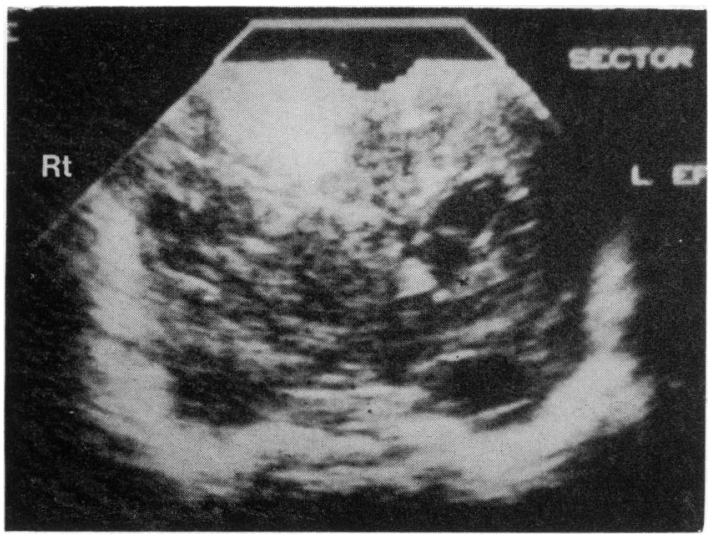

Fig. 3 Infant in case 2-Coronal scan at 5 days showing shrinkage and increased echodensity of right sided mass.

resolution of midline shift and contralateral ventricular dilatation. The mass later disappeared but slight ventricular dilatation persisted. These appearances may represent subarachnoid or possibly subdural haematoma with compression of underlying brain tissue and late cerebral atrophy.

\section{Case 3}

A boy was delivered vaginally, at term, and his birthweight was $2.48 \mathrm{~kg}$. Multiple bruises developed over 12 hours. Investigations showed thrombocytopaenia only, platelets $20000 / \mathrm{mm}^{3}\left(20 \times 10^{9} / 1\right)$ at lowest. Maternal serum contained antiplatelet antibodies. From 12 hours left sided twitching developed, persisting intermittently for 4 days. At 48 hours he was transferred to the regional neonatal unit. Subdural aspiration through the coronal suture was performed and $9 \mathrm{ml}$ bloody fluid removed. No further seizures occurred, but the infant's behaviour was abnormal on discharge at 18 days. At 6 months his development was normal.
Ultrasound findings. At 48 hours, an echodense mass filled the right temporoparietal region, dimensions $2 \times 2 \times 3 \mathrm{cms}$ (Fig. 4). There was associated midline shift to the left with dilatation of the left lateral ventricle. Irregularities at the medial margin suggested intracerebral extension of a subdural haematoma. In subsequent scans, midline shift resolved and the mass disappeared.

\section{Case 4}

A boy was delivered by emergency caesarean section for fetal distress at 32 weeks, his birthweight was $2.05 \mathrm{~kg}$. There was vaginal bleeding from the placenta praevia from 20 weeks onwards. The infant was intubated from birth and his Apgar score was 3 at 1 minute. He was ventilated because of persistent cyanosis related to persistent fetal circulation. There was a good response to tolazoline, which was infused from 12 hours to 8 days. On day 5 , serum bilirubin was $362 \mu \mathrm{mol} / 1(20 \mathrm{mg} / 100 \mathrm{ml})$; double volume exchange transfusion was performed through an umbilical arterial catheter. No complications were noted and he was extubated on day 11 . Recovery was complicated by Escherichia coli septicaemia associated with episodes of apnoea on
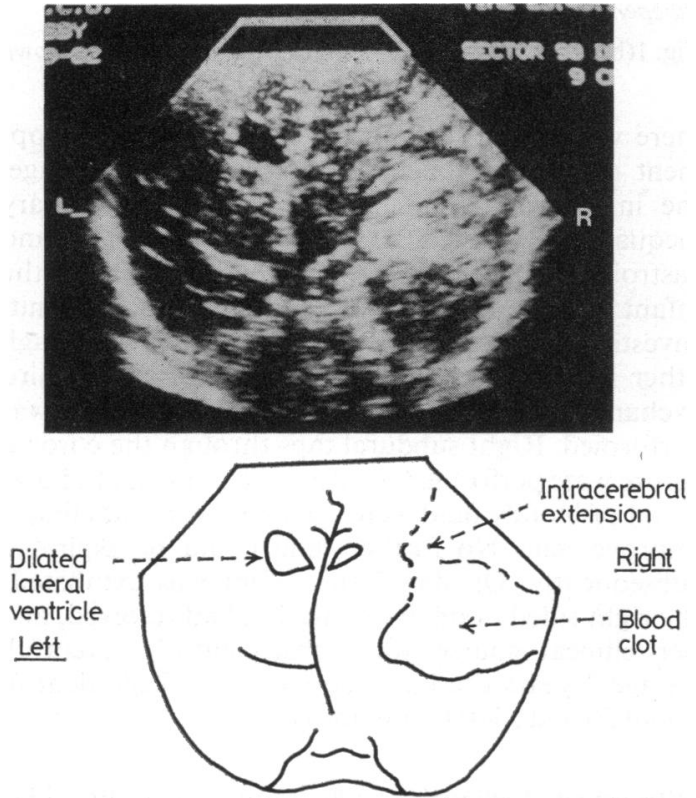

Fig. 4 Infant in case 3-Coronal scan at 48 hours showing echodense mass filling right temporoparietal region with midline displacement and dilatation of left lateral ventricle. 
day 24. Recovery after treatment was uneventful. Follow up was normal at 2 months.

Ultrasound findings. Ultrasound scans were normal on days 1,4 , and 6 . A routine scan on day 10 showed an echodense mass over the right frontoparietal region associated with midline shift to the left (Fig. 5). Two weeks later the midline had returned to normal, the mass was shrinking, but moderate bilateral ventricular dilation was evident. This persisted at 2 months without development of progressive hydrocephalus. This appearance suggested subarachnoid haematoma.

\section{Case 5}

A girl was delivered unattended at home at 28 weeks' gestation, birthweight $900 \mathrm{~g}$. Initial problems included birth asphyxia, hypothermia to $30^{\circ} \mathrm{C}$, and severe acidosis. There was no respiratory distress The infant was ventilated from hospital admision at one hour until 12 hours. At 7 days a profound acidosis with rapidly developing anaemia occurred. A two volume exchange transfusion for presumed septicaemia was performed through an umbilical arterial catheter. Intracranial haemorrhage was subsequently diagnosed after convulsions developed. Subdural tap resulted in the aspiration of $7 \mathrm{ml}$ of bloody fluid. Disseminated coagulopathy was

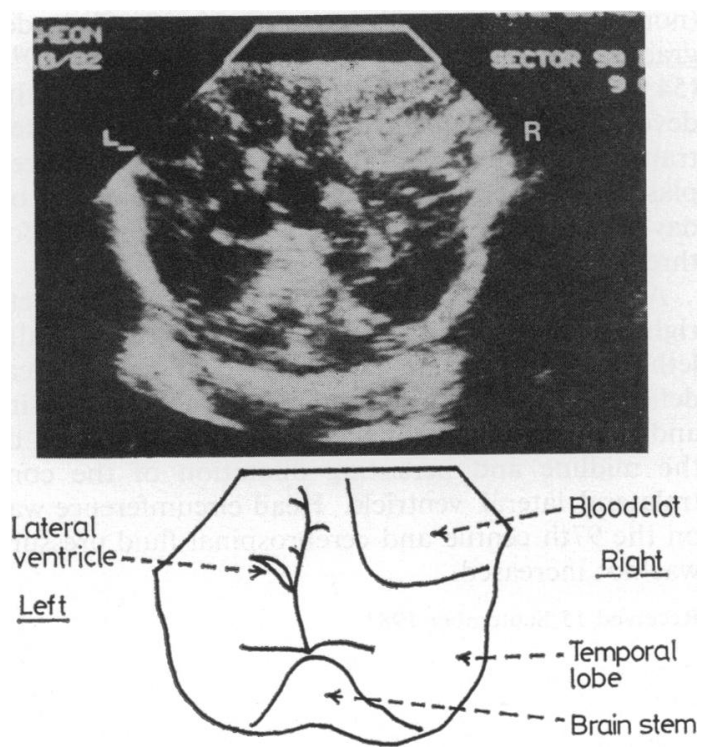

Fig. 5 Infant in case 4-Coronal scan showing echoreflectant mass displacing right parietal lobe. The right lateral ventricle is not seen. unresponsive to medical management. The infant died at 8 days. Klebsiella oxytoca was grown from blood cultures taken 24 hours before death. Necropsy permission was refused.

Ultrasound findings. The initial ultrasound scans were normal. At 7 days, a left sided echolucent mass $1.5 \times 1 \mathrm{~cm}$ in diameter, was evident with midline shift to the right and intraventricular haemorrhage (Grade III) bilaterally. The edges of the mass were indeterminate, perhaps representing intracerebral extension of subdural haemorrhage.

\section{Discussion}

Secondary haemorrhagic disease of the newborn leading to fatal intracranial haemorrhage of a characteristic type has been described by Chessells et al. ${ }^{2}$ Subarachnoid haematoma with varying degrees of infiltration and destruction of underlying brain follows venous rupture after disseminated coagulation failure, and leads to death. Asphyxia, shock, and acidosis are leading precipitating factors. ${ }^{56}$ Coagulopathy and intracranial haemorrhage have also had a long association with rhesus incompatibility in the neonate. ${ }^{7}$

Chessells comments that the logical management of such infants would be exchange transfusion before development of haemorrhage. Larroche, ${ }^{1}$ however, describing 33 cases of subarachnoid haematoma found in 1000 necropsies, noted that 20 infants had had exchange transfusion performed for varied clinical indications, and postulated that exchange transfusion itself might, via transient venous pressure rises, cause subarachnoid haemorrhage with or without underlying venous infarction of the brain-the origin of bleeding being the delicate leptomeningeal plexus. Bada et al. ${ }^{8}$ confirmed associated intracranial pressure changes during exchange transfusion by fontogram recording. In preterm infants cerebral blood flow rises and falls passively with mean arterial blood pressure.

The presence of intracranial clot at necropsy, despite antemortem coagulation failure, is explicable if the site of haemorrhage is subpial, on the basis of adjacent high brain thromboplastin values. The prognosis relates to damage to underlying brain, the subarachnoid haematoma itself often resolving without trace. ${ }^{1}$

The infants described here represent extracerebral haemorrhage after coagulopathy, asphyxia, anoxia, or hypothermia. It is not possible from ultrasound scan to define precisely the site of origin of haemorrhage, however, the clinical course follows closely the extent of visible ultrasonic abnormality. 
Infant 1 , who had a large lesion without evident intracerebral extension, had no neonatal neurological signs and no apparent sequelae. Where clinical signs resulted from cerebral compression (infants 2,3 , and 5), persisting cerebral abnormality was evident on ultrasound, and clinically.

In four of five infants, development of haemorrhage followed within one to two days of exchange transfusion. In all these infants there was a history of perinatal asphyxia, with or without complicating later hypoxaemia or hypothermia.

It would seem useful, therefore, if infants with a history of asphyxia having exchange transfusions in the newborn period had ultrasound scans performed after the procedure, and 3-4 days thereafter. The incidence of intracranial haemorrhage after this procedure may be higher than previously recognised, clinical signs being evident in a minority of cases only.

\section{References}

1 Larroche J-C. Developmental pathology of the neonate. Amsterdam and New York: Excepta Medica, 1977.

2 Chessells JM, Wigglesworth JS. Secondary haemorrhagic disease of the newborn. Arch Dis Child 1970;45:539-43.

3 Pape KE, Wigglesworth JS. Haemorrhage, ischaemia and the perinatal brain. (Clinics in Developmental Medicine 1979; 69/70) London: SIMP, 1979.

4 Cooke RWI. Ultrasound examination of neonatal heads (letter). Lancet 1979 ;ii:38.

5 Towbin A. Central nervous system damage in the human fetus and newborn infant. Am J Dis Child 1970;119: $529-42$.

- Fenichel GM. Neonatal neurology. New York: Churchill Livingstone, 1980:99-100.

7 Leonard MF. Hemolytic disease of the newborn (Erythroblastosis fetalis). J Pediatr 1945;27:249-65.

8 Bada HS, Chua C, Salmon JH, Hajjar W. Changes in intracranial pressure during exchange transfusion. J Pediatr 1979;94:129-32.

Correspondence to Dr M E I Morgan, Department of Child Health, Alder Hey Children's Hospital, Eaton Road, Liverpool L12 2AP.

Received 13 June 1983

\section{Addendum}

Since acceptance of the above article, we have observed a further case of subarachnoid haematoma after exchange transfusion in a rhesus isoimmunised infant.

\section{Case report}

A rhesus isoimmunised boy was delivered by emergency caesarean section at 36 weeks; birthweight $3.12 \mathrm{~kg}$. At birth he was asphyxiated and pale, with hepatosplenomegaly $(3 \mathrm{~cm})$. He was immediately intubated, ventilated, and 2 volume exchange transfusion performed through an umbilical venous line. Cord haemoglobin was $5.3 \mathrm{~g} / \mathrm{dl}$. At transfer to the regional unit at 10 hours of age, an ultrasound scan showed a large right temporal blood clot with contralateral displacement of the midline. Coagulation screening at this point was abnormal- prothrombin time $32 \mathrm{sec}$, British comparative ratio $2 \cdot 7$, activated partial thromboplastin time $52 \mathrm{sec}$ (normal $30-40 \mathrm{sec}$ ), fibrinogen $1.22 \mathrm{~g} / \mathrm{l}$, fibrin degradation products $10-40 \mu \mathrm{g} / \mathrm{ml}$, platelets $54 \times 10^{9} / 1$ $\left(54000 / \mathrm{mm}^{3}\right)$. A consumption coagulopathy developed subsequently, managed by repeated transfusion of platelet concentrate and fresh frozen plasma. Generalised convulsions developed on day 2 and were managed by phenobarbitone for three weeks.

At one month of age the infant had a complete right sided 3rd nerve palsy, and was generally lethargic. There were no other focal neurological deficits. Ultrasound showed decreased echodensity and slight shrinkage of the clot, with restoration of the midline and persisting dilatation of the contralateral lateral ventricle. Head circumference was on the 97th centile and cerebrospinal fluid pressure was not increased.

Received 15 September 1983 